\title{
Simultaneous Tests for Homogeneity of Two Zero-inflated (Beta) Populations
}

Supplemental file

\author{
Luna Sun and Alix I. Gitelman \\ Department of Statistics, Oregon State University
}

Result 1 (Partition of multivariate normal distribution (Seely's Notes, unpublished)). Suppose $Y \sim$ $N_{p}(\mu, V)$ where $V$ is nonsingular. Let $Y, \mu$ and $V$ be similarly partitioned in the form $Y=\left[\begin{array}{l}Y_{1} \\ Y_{2}\end{array}\right]$, $\mu=\left[\begin{array}{l}\mu_{1} \\ \mu_{2}\end{array}\right], V=\left[\begin{array}{ll}V_{11} & V_{12} \\ V_{21} & V_{22}\end{array}\right]$. Assume $Y_{1}$ is $q \times 1$ and that $s=p-q$ so that $Y_{2}$ is $s \times 1$. Set $V_{11.2}=$ $V_{11}-V_{12} V_{22}^{-1} V_{21}$ and let $T=Y_{1}-V_{12} V_{22}^{-1} Y_{2}$. Then

1. $T \sim N_{q}\left(\mu_{1}-V_{12} V_{22}^{-1} \mu_{2}, V_{11.2}\right)$ and $V_{11.2}$ is nonsingular.

2. $T$ and $Y_{2}$ are independent random vectors.

Theorem 1 (Asymptotic Independence among Score Tests). $\hat{S_{1}}, \hat{S_{2}}$ and $\hat{S_{3}}$ (and also $\tilde{S_{1}}, \tilde{S_{2}}$ and $\tilde{S_{3}}$ ) are asymptotically independent.

Proof. Consider $\hat{S_{1}}, \hat{S_{2}}$ and $\hat{S_{3}}$ first: Denote

$$
\begin{gathered}
\omega=(p, \mu, \phi)^{\prime}, \zeta=(\delta, p, \mu, \phi)^{\prime}=\left(\delta, \omega^{\prime}\right)^{\prime}, \eta=(\gamma, \delta, p, \mu, \phi)^{\prime}=\left(\gamma, \zeta^{\prime}\right)^{\prime}=\left(\gamma, \delta, \omega^{\prime}\right)^{\prime}, \\
\theta=(\beta, \gamma, \delta, p, \mu, \phi)^{\prime}=\left(\beta, \eta^{\prime}\right)^{\prime}=\left(\beta, \gamma, \zeta^{\prime}\right)^{\prime}=\left(\beta, \gamma, \delta, \omega^{\prime}\right)^{\prime} .
\end{gathered}
$$

Recall that

$$
\begin{aligned}
& \hat{S_{1}}={\hat{s_{1}}}^{\prime}\left(\hat{A_{1}}-\hat{C_{1}}{\hat{D_{1}}}^{-1}{\hat{C_{1}}}^{\prime}\right)^{-1} \hat{s_{1}}, \\
& \hat{S_{2}}={\hat{s_{2}}}^{\prime}\left(\hat{A_{2}}-\hat{C_{2}}{\hat{D_{2}}}^{-1}{\hat{C_{2}}}^{\prime}\right)^{-1} \hat{s_{2}}
\end{aligned}
$$

and

$$
\hat{S_{3}}={\hat{s_{3}}}^{\prime}\left(\hat{A_{3}}-{\hat{C_{3}}}_{\hat{D}_{3}}^{-1}{\hat{C_{3}}}^{\prime}\right)^{-1} \hat{s_{3}}
$$

Under $H_{0}$,

$$
s_{1}=\frac{\partial l_{1 f}}{\partial \delta}=\frac{\partial l_{f}}{\partial \delta}, s_{2}=\frac{\partial l_{2 f}}{\partial \gamma}=\frac{\partial l_{f}}{\partial \gamma}, \text { and } s_{3}=\frac{\partial l_{3 f}}{\partial \beta}=\frac{\partial l_{f}}{\partial \beta}
$$


And

$$
\hat{s_{1}}=s_{1}(\hat{\omega}), \hat{s_{2}}=s_{2}(\hat{\zeta}), \hat{s_{3}}=s_{3}(\hat{\eta})
$$

where $\hat{\omega}, \hat{\zeta}$ and $\hat{\eta}$ are maximum likelihood estimates of $\omega$ under $H_{0}^{\prime}, \zeta$ under $H_{0}^{\prime \prime}$ and $\eta$ under $H_{0}^{\prime \prime \prime}$, respectively (i.e., $\hat{s_{1}}=\left.\frac{\partial l_{f}}{\partial \delta}\right|_{\omega=\hat{\omega}}, \hat{s_{2}}=\left.\frac{\partial l_{f}}{\partial \gamma}\right|_{\zeta=\hat{\zeta}}$ and $\hat{s_{3}}=\left.\frac{\partial l_{f}}{\partial \beta}\right|_{\eta=\hat{\eta}}$ ). Notice that when $H_{0}$ is true, $H_{0}^{\prime}$, $H_{0}^{\prime \prime}$ and $H_{0}^{\prime \prime \prime}$ are all true. Expanding $\hat{s_{1}}, \hat{s_{2}}$ and $\hat{s_{3}}$ around the true parameters $\theta_{0}$ via Taylor expansion with $l_{f}=l$ :

$$
\begin{gathered}
\hat{s_{1}}=\frac{\partial l}{\partial \delta}-I_{\delta \omega_{0}} I_{\omega_{0} \omega_{0}}^{-1} \frac{\partial l}{\partial \omega_{0}}+\mathscr{O}_{p}(1), \\
\hat{s_{2}}=\frac{\partial l}{\partial \gamma}-I_{\gamma \zeta_{0}} I_{\zeta_{0} \zeta_{0}}^{-1} \frac{\partial l}{\partial \zeta_{0}}+\mathscr{O}_{p}(1)
\end{gathered}
$$

and

$$
\hat{s_{3}}=\frac{\partial l}{\partial \beta}-I_{\beta \eta_{0}} I_{\eta_{0}}^{-1} \eta_{0} \frac{\partial l}{\partial \eta_{0}}+\mathscr{O}_{p}(1)
$$

where

$$
\begin{aligned}
& I_{\delta \omega_{0}}=E\left(-\frac{\partial^{2} l}{\partial \delta \partial \omega^{T}} \mid H_{0}\right), I_{\omega_{0} \omega_{0}}=E\left(-\frac{\partial^{2} l}{\partial \omega \partial \omega^{T}} \mid H_{0}\right), I_{\gamma \zeta_{0}}=E\left(-\frac{\partial^{2} l}{\partial \gamma \partial \zeta^{T}} \mid H_{0}\right), \\
& I_{\zeta_{0} \zeta_{0}}=E\left(-\frac{\partial^{2} l}{\partial \zeta \partial \zeta^{T}} \mid H_{0}\right), I_{\beta \eta_{0}}=E\left(-\frac{\partial^{2} l}{\partial \beta \partial \eta^{T}} \mid H_{0}\right), I_{\eta_{0} \eta_{0}}=E\left(-\frac{\partial^{2} l}{\partial \eta \partial \eta^{T}} \mid H_{0}\right),
\end{aligned}
$$

and

$$
\frac{\partial l}{\partial \omega_{0}}=\left.\frac{\partial l}{\partial \omega}\right|_{\omega=\omega_{0}}, \frac{\partial l}{\partial \zeta_{0}}=\left.\frac{\partial l}{\partial \zeta}\right|_{\zeta=\zeta_{0}}, \frac{\partial l}{\partial \eta_{0}}=\left.\frac{\partial l}{\partial \eta}\right|_{\eta=\eta_{0}} .
$$

Let

$$
s_{01}=\frac{\partial l}{\partial \delta}-I_{\delta \omega_{0}} I_{\omega_{0} \omega_{0}}^{-1} \frac{\partial l}{\partial \omega_{0}}, s_{02}=\frac{\partial l}{\partial \gamma}-I_{\gamma \zeta_{0}} I_{\zeta_{0} \zeta_{0}}^{-1} \frac{\partial l}{\partial \zeta_{0}} \text { and } s_{03}=\frac{\partial l}{\partial \beta}-I_{\beta} \eta_{0} I_{\eta_{0} \eta_{0}}^{-1} \frac{\partial l}{\partial \eta_{0}}
$$

Since the score function can be written as

$$
\frac{\partial l}{\partial \theta}=\left(\begin{array}{c}
\frac{\partial l}{\partial \beta} \\
\frac{\partial l}{\partial \gamma} \\
\frac{\partial l}{\partial \delta} \\
\frac{\partial l}{\partial p} \\
\frac{\partial l}{\partial \mu} \\
\frac{\partial l}{\partial \phi}
\end{array}\right)=\left(\begin{array}{c}
\frac{\partial l}{\partial \beta} \\
\frac{\partial l}{\partial \eta}
\end{array}\right)=\left(\begin{array}{c}
\frac{\partial l}{\partial \beta} \\
\frac{\partial l}{\partial \gamma} \\
\frac{\partial l}{\partial \zeta}
\end{array}\right)=\left(\begin{array}{c}
\frac{\partial l}{\partial \beta} \\
\frac{\partial l}{\partial \gamma} \\
\frac{\partial l}{\partial \delta} \\
\frac{\partial l}{\partial \omega}
\end{array}\right),
$$


we have $\frac{\partial l}{\partial \theta} \stackrel{d}{\rightarrow} N\left(0, I\left(\theta_{0}\right)\right)$. Or

$$
\left(\begin{array}{c}
\frac{\partial l}{\partial \beta} \\
\frac{\partial l}{\partial \eta}
\end{array}\right) \stackrel{d}{\rightarrow} N\left(\left(\begin{array}{c}
0 \\
0
\end{array}\right),-E\left(\begin{array}{cc|c}
\frac{\partial^{2} l}{\partial \beta^{2}} & \frac{\partial^{2} l}{\partial \beta \partial \eta^{T}} \\
\frac{\partial^{2} l}{\partial \eta \partial \beta} & \frac{\partial^{2} l}{\partial \eta \partial \eta^{T}}
\end{array} \mid H_{0}\right)\right) .
$$

As the sample size going to $+\infty$, using the result from partition of multivariate normal distribution, under $H_{0}, s_{03}$ is independent of $\frac{\partial l}{\partial \eta}=\left(\begin{array}{c}\frac{\partial l}{\partial \gamma} \\ \frac{\partial l}{\partial \zeta}\end{array}\right)=\left(\begin{array}{c}\frac{\partial l}{\partial \gamma} \\ \frac{\partial l}{\partial \delta} \\ \frac{\partial l}{\partial \omega}\end{array}\right)$. Since $s_{01}$ and $s_{02}$ are only functions of $\left(\frac{\partial l}{\partial \gamma}, \frac{\partial l}{\partial \zeta}\right)$ and $\left(\frac{\partial l}{\partial \delta}, \frac{\partial l}{\partial \omega}\right), s_{03}$ is independent of $s_{01}$ and $s_{02}$. Similarly, it is easy to show $s_{01}$ and $s_{02}$ are independent as well.

For the independence among $\tilde{S_{1}}, \tilde{S_{2}}$ and $\tilde{S_{3}}$, similar arguments can be made.

Theorem 2 (Size of Hybrid test). The hypothesis in (8) is

$$
H_{0}: p_{1}=p_{2} \text { and } h_{Y_{1}}=h_{Y_{2}} \text { vs } H_{1}: \text { Either equality fails. }
$$

Here, $p_{1}$ and $p_{2}$ are the population zero proportions for populations 1 and 2 , and $h_{Y_{1}}$ and $h_{Y_{2}}$ are the pdffor the non-zero components of populations 1 and 2. To test these hypotheses at the level $\alpha$, the Hybrid procedure involves the following steps:

1. Test $H_{01}: p_{1}=p_{2}$ versus $H_{11}: p_{1} \neq p_{2}$.

2. $H_{01}$ is rejected if the $p$-value is $p<\alpha_{1}$. In this case, reject $H_{0}$ in (8); otherwise, test $H_{02}$ : $h_{Y_{1}}=h_{Y_{2}}$ versus $H_{12}: h_{Y_{1}} \neq h_{Y_{2}}$.

3. $H_{02}$ is rejected if the $p$-value is $p<\alpha_{2}$. In this case, reject $H_{0}$ in (8); otherwise, do not reject $\mathrm{H}_{0}$.

The size of Hybrid is less than or equal to $\alpha_{1}+\alpha_{2}$.

Proof. Define

$$
A=\left\{\left(p_{1}, p_{2}\right) \in(0,1)^{2}: p_{1}=p_{2}\right\} \text { and } B=\left\{h_{Y_{1}}, h_{Y_{2}} \in \mathscr{F}: h_{Y_{1}}=h_{Y_{2}}\right\}
$$

where $\mathscr{F}$ is the space of a particular family of pdf. Under the set notation, $H_{0}, H_{1}, H_{01}, H_{11}, H_{02}$ and $H_{12}$ can be expressed as $H_{0}: A \cap B, H_{1}: A^{c} \cup B^{c}, H_{01}: A, H_{11}: A^{c}, H_{02}: A \cap B$ and $H_{12}: A \cap B^{c}$. Further define $\mathfrak{R}_{1}, \mathfrak{R}_{2}$ and $\mathfrak{R}$ as rejection region for $H_{01}, H_{02}$ and $H_{0}$, then

$$
C=\left\{\mathbf{x}: \mathbf{x} \in \mathfrak{R}_{1}\right\} \text { and } D=\left\{\mathbf{x}: \mathbf{x} \in \mathfrak{R}_{2}\right\},
$$


then we have

$$
C \cup\left(C^{c} \cap D\right)=\{\mathbf{x}: \mathbf{x} \in \mathfrak{R}\} .
$$

It is reasonable to assume $A$ and $B$ are independent because the form of $h_{Y}$ does not depend on $p$, neither the other way around. Also, it is reasonable to assume $B$ and $C$ are independent because $C$ only concerns the $p$ 's.

Let $\alpha_{0}, \alpha_{1}$ and $\alpha_{2}$ denote the type I error rates of $H_{0}$ vs $H_{1}, H_{01}$ vs $H_{11}$ and $H_{02}$ vs $H_{12}$, respectively.

Then

$$
\begin{gathered}
\alpha_{0}=P\left(\text { Reject } H_{0} \mid H_{0} \text { is true }\right)=P\left(C \cup\left(C^{C} \cap D\right) \mid A \cap B\right), \\
\alpha_{1}=P(C \mid A) \text { and } \alpha_{2}=P(D \mid A \cap B) .
\end{gathered}
$$

Since $C \cap\left(C^{c} \cap D\right)=\emptyset$, we have $(C \mid A \cap B) \cap\left(C^{c} \cap D \mid A \cap B\right)=\emptyset$. Therefore,

$$
P\left(C \cup\left(C^{c} \cap D\right) \mid A \cap B\right)=P(C \mid A \cap B)+P\left(\left(C^{c} \cap D\right) \mid A \cap B\right) .
$$

Since $C^{c} \cap D \subset D$, there is $C^{c} \cap D|A \cap B \subset D| A \cap B$. Therefore,

$$
\begin{gathered}
P\left(\left(C^{C} \cap D\right) \mid A \cap B\right) \leq P(D \mid A \cap B)=\alpha_{2} . \\
P(C \mid A \cap B)=\frac{P(C \cap A \cap B)}{P(A \cap B)}=\frac{P(B \mid A \cap C) \cdot P(A \cap C)}{P(B \mid A) \cdot P(A)}=P(C \mid A) \cdot \frac{P(B \mid A \cap C)}{P(B \mid A)}
\end{gathered}
$$

Because we assume $A$ and $B$ are independent and $B$ and $C$ are independent, $P(B \mid A)=P(B)$ and $P(B \mid A \cap C)=P(B)$. Hence,

$$
P(C \mid A \cap B)=P(C \mid A)=\alpha_{1}
$$

As a result,

$$
\alpha_{0}=P\left(C \cup\left(C^{c} \cap D\right) \mid A \cap B\right)=P(C \mid A \cap B)+P\left(\left(C^{c} \cap D\right) \mid A \cap B\right) \leq \alpha_{1}+\alpha_{2} .
$$

Under the Bonferroni correction, $\alpha_{1}=\alpha_{2}=\alpha / 2$.

\section{Reference}

Justus Seely. Linear Model Theory Notes, unpublished. Department of Statistics, Oregon State University. 